\title{
Clinical Guidelines, Closing Gaps between the National Context,
}

\section{and the Global trends}

Hussain $\mathrm{YH}^{*}$

Dubai Health Authority, Dubai

*Corresponding author: Hussain YH, Dubai Health Authority, and Tel:

+995599957328; Email: hussainh569@gmail.com

\section{Short communication}

Evidenced-based and national context orientated Guidelines help clinicians translate best evidence into best practice. A well-calipered guideline improves quality by reducing variations in healthcare delivery. Clinical Guidelines remarkably contributing to Improving diagnostic accuracy, enhancing effective therapy, and restricting ineffective - or potentially non-effective interventions. Clinical guidelines methodology seems to be poorly defined and clearly varies at different organizations. One of the gaps needs to be closed under national clinical guidelines is to generate and develop a quality-driven, evidence-based through utilizing an efficient and rigors methodology for action and setting up ready recommendations with multi-disciplinary applicability. The Process of development, that permit moving from conception to completion in a well-defined period of time, always emphasizes a logical sequence of key action statements supported by amplifying text, evidence profiles, as well as recommendations which link action to evidence.

Keywords: Clinical guidelines; Methodology

\section{What should National Clinical Guidelines Worth to:}

National Clinical Guidelines should be Quality linked structured e.g. addressing quality improvement at the forefront of guideline creation, through enrolling the best evidence, as well as multidisciplinary consensus in order, prioritize recommendations. National Guidelines shall be looking powerful if it is driven by the EVIDENCE-BASED approach, e.g. Supporting all decisions with the best available research evidence identified by systematic literature review.
Ensuring an EFFICIENCY of national guidelines through maximum utilization of all available resources to initiate a timely product, and stepping from conception to publication within an assigned period of time.

Rigorous METHODOLOGY and transparent manipulation is recommended, reproducible, and needs to be applied in a consistent way to support user linking recommendations to communicating the level of evidence, understanding benefit-harm-cost relationship, and identifying the roles of values and patient preferences in a process of decision making.

Highlighting obviously the Action-ready recommendations, which is telling providers what to do, under what specific circumstance, and to whom by following an unambiguous procedure and literature that facilitates implementation and measurement.

Validating national Guidelines through multidisciplinary applicability and validity, and enables all stakeholders (e.g., specialists, primary care, nursing, allied health, consumers) should be always considered as part of the development and implementation processes.

Due to huge variability in guideline development methodology, between different organizations or within the same organization, it has been experienced that, mandates for a systematic approach to guideline development must be addressed. In spite of a plethora of techniques revealed in published guidelines, it is always hard to find a single, comprehensive "how-to" manual with a valid and pragmatic approach that could be readily implemented. Decisive questions have to be steering the directions of the final product of the Guidelines e.g. Why guidelines control and governing quality care, what 


\section{Journal of Quality in Healthcare \& Economics}

makes a guideline valid and useful? What Principles, steps, and processes are essentials for of guideline development, Identifying, sorting out and utilizing best published evidence, prioritizing opportunities to quality improvement, Consciously Understanding main action statements: recognized to be the backbone of a clear and usable guideline, recognizing evidence profiles and how it looks: which contribute to promote transparency. Grading of recommendation: the link between action and evidence. Evaluating Guild line implementation: Tracing an opportunities to influence clinician behaviour.

Experience to Guideline development varies when the organization is still new to generating guideline, lots of benefit from understanding the complexities and nature will be yielded, process outlining approach can be followed as a starting point for their own efforts. Yet, an established guideline development process may wish to compare their current processes to those described, to identifying numbers of areas for improved quality, efficiency, and or both. Guideline panel's members and working groups can easily derive greater insight and understanding of development methodology, and allowing them to contribute most effectively. Staff who are supporting the guideline development will find practical suggestions on staying focused and efficient and may consider using, or adapting, different manual as well as a template for their own processes.

\section{Quality guidelines}

Adhering to Purpose and scope: Describing guideline objective(s), Covering clinical question(s), and the target of Guideline implementation.

Enrolling main stakeholders: Through identifying individuals from all concerned professional groups; patients' intended users to be clearly defined; as well as target guideline to be piloted among target users.

Scientifically solid development: adopting the Systematic methodology, through evidence searching strategy. An Approach formulating recommendations must be clearly addressed; indicating health benefits, side effects, as well as risks; the guideline must be revised by experts in advance to publication; and guidelines updating procedure is provided.

Guideline clarity: management options are clear Recommendations are identifiable; and the guideline is supported with tools for application.

Applicability: through developing criteria for monitoring and/or audit purposes.

Independence of Editorial Board: Addressing funding body interest and staying away of influencing final recommendations needs to be secured; identifying potential conflicts of interest.

Concerned Organizations who showed such interest must have sold production strategies that put in consideration a rigor and pragmatism, as clinical guidelines extremely important as a key metric of quality healthcare, clinicians mandating realize how they are best utilized to improve services and health care.

\section{References}

1. Institute of Medicine. Clinical Practice Guidelines We Can Trust. Washington, DC. National Academies Press. 2011.

2. IOM (Institute of Medicine). Finding What Works in Health Care: Standards for Systematic Reviews. Washington, DC. National Academies Press. 2011.

3. Agency of Healthcare Research and Quality. Methods guide for effectiveness and comparative effectiveness reviews. Rockville, MD: Agency for Healthcare Research and Quality; 2014. AHRQ Publication No. 10(14)-EHC063-EF.

4. Norris SL, Holmer HK, Burda BU, Ogden LA, Fu R (2012) Conflict of interest policies for organizations producing a large number of clinical practice guidelines. PLoS One 7(5): e375413. 